\title{
Mobile robotic fabrication beyond factory conditions: case study Mesh Mould wall of the DFAB HOUSE
}

\section{Journal Article}

Author(s):

Dörfler, Kathrin (D); Hack, Norman; Sandy, Timothy; Giftthaler, Markus (D); Lussi, Manuel; Walzer, Alexander N. (D); Buchli, Jonas; Gramazio, Fabio; Kohler, Matthias

Publication date:

2019-12

Permanent link:

https://doi.org/10.3929/ethz-b-000407460

Rights / license:

In Copyright - Non-Commercial Use Permitted

Originally published in:

Construction Robotics 3(1-4), https://doi.org/10.1007/s41693-019-00020-w 


\title{
Mobile robotic fabrication beyond factory conditions: Case study Mesh Mould wall of the DFAB HOUSE
}

\author{
Kathrin Dörfler • Norman Hack • \\ Timothy Sandy • Markus Giftthaler • \\ Manuel Lussi • Alexander N. Walzer • \\ Jonas Buchli • Fabio Gramazio • \\ Matthias Kohler
}

Received: 10.02.2019 / Accepted: 25.6.2019

\begin{abstract}
The development of novel robotic fabrication technologies in architecture concentrates largely on integrating stationary industrial-type robots into off-site prefabrication processes. By contrast, few enabling robotic technologies exist today that allow robotic fabrication processes to be mobile and implemented directly on building sites. While mobile in-situ fabrication offers a large range of architectural potentials, its realization requires to address fundamental challenges. First, the production of large-scale and potentially monolithic structures on-site requires an advanced robotic fabrication system that can fulfill the material, structural- and architectural-related demands associated with it. Second, the poorly structured nature of building sites requires mobile robotic systems to be equipped with advanced sensing and control solutions to contend with uncertain conditions found on-site. The research discussed in this paper addresses both of these subjects. It applies a novel construction system for non-standard reinforced concrete structures, termed Mesh Mould, to explore the fabrication of large-scale and monolithic building structures using a mobile robot on site. It further investigates sensorintegrated adaptive fabrication strategies to achieve the accurate fabrication of such a large-scale structure, and this is done despite prevalent uncertainties related to the building site environment, the mobile robotic system, and the material behavior during fabrication. The results of this research were realized in a slender, doubly curved, reinforced concrete wall at the DFAB HOUSE at NEST. This research demonstrator provides the unique opportunity to present robotic in situ fabrication not merely as a future possibility, but as a reality applied to a tangible construction project.
\end{abstract}

Keywords Digital fabrication · Construction robotics · In situ fabrication · Adaptive fabrication · Mobile manipulation

This research was supported by Swiss National Science Foundation through the NCCR Digital Fabrication (NCCR Digital Fabrication Agreement 51NF40-141853) and a Professorship Award to Jonas Buchli (Agreement PP00P2 $2_{1} 38920$ ). 
1 Introduction

Robotic technologies for novel additive fabrication processes at a full architectural scale enable a higher degree of freedom in design possibilities and geometric differentiation, functional integration, and integrated design-to-fabrication workflows $[28,22,29]$. Hence, they promise to yield a multitude of benefits to the construction industry for improving the productivity, quality, and sustainability of building construction [39]. However, the advancement of robotic fabrication has mostly been focused on integrating industrial-type robots into dedicated construction factories for the off-site prefabrication of building components. Some prominent and recent examples are the Swiss construction company ERNE AG Holzbau [7], which used a large-scale gantry robot for the prefabrication of bespoke timber components for a roof construction in 2015 [51] (Fig. 1(a)), or the Gramazio Kohler Research group at ETH Zurich, who prefabricated wooden frame modules for a residential unit with the gantry robot of the Robotic Fabrication Laboratory in 2018 [20,47]. Construction factories facilitate the implementation of automated prefabrication processes on many levels, one of the most important factors being the ability to provide a controlled and fully structured environment. Prefabrication also corresponds to the modernist tradition that promotes the separation of functions implicit in the architectural components (e.g., the divisions between structure and skin in curtain wall constructions, or the separation among walls, columns, and slabs as found in the plattenbau - an architecture that is conveniently massproduced and assembled) $[24,32,42]$. The inherent modularization of prefabrication is essentially congruent with the manufacturing industry's traditional methods of mass production, and therefore provides many benefits in the context of robotics and automation [24,32]. Beyond these benefits, however, prefabrication imposes also a number of limitations in the architectural and material-related realms. Prefabricated components must comply with transportation directives regarding size and weight, and they must sustain the forces acting on them during transportation, lifting, and final assembly. Components that cannot sustain these forces may require temporary bracing; this applies to prefabricated structures such as masonry walls, which must be equipped with vertical channels for anchor bars that enable their transport and erection [36]. With respect to joining, the final assembly of components on site poses the challenge of high-accuracy fabrication requirements, since some potentially necessary adjustments can only occur in the connections and in the joints between components. Furthermore, these components must be joined in such a way that the connections ensure the structural integrity of the whole structure. Lastly, the joining of such prefabricated components into the fullscale building structure on site is still dependent on manual labor (Fig. 1(b)). Consequently, these final manual manufacturing steps interrupt the digital process chain from design to fabrication [31]. 


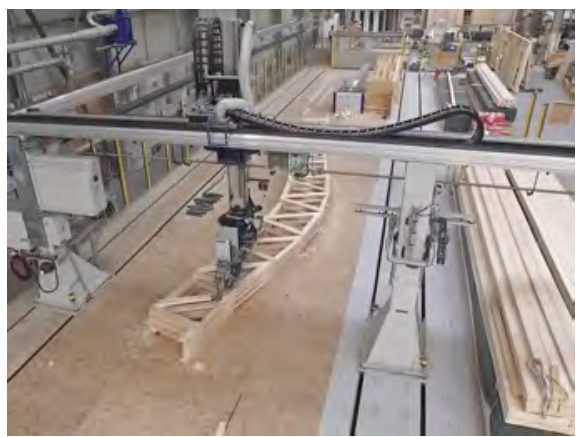

(a)

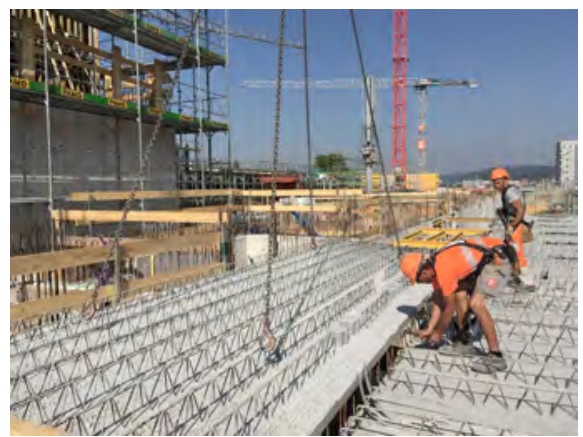

(b)

Fig. 1 (a) Example of a robotic prefabrication process in a dedicated off-site construction factory: The gantry robot at ERNE AG Holzbau fabricates a bespoke timber module, which is a building component of a large-scale roof construction (2015) [7]. (b) Example of a manual assembly process on a building site: Construction workers assemble a concrete composite floor plate system with lattice girders, PJZ Zurich building site (2018).

Despite these limitations, few enabling robotic technologies currently exist that would allow robotic systems to be integrated into in situ ${ }^{1}$ construction processes, and thereby introduce new possibilities to architectural design and building construction. Since an in situ fabricated building structure is subject to fewer constraints on the scale of the objects and the material system used, it allows for the production of structures designed to be free of components and modularization [42]. For example, this is seen as required in structures with high dynamic and alternating loads, which must be monolithic and continuous to be structurally sound; examples of such structures are cast-in-place reinforced concrete structures [18]. As an alternative approach to the compositional and modular design logic, additive robotic in situ processes could provide different design options to achieve architectural differentiation with a higher degree of continuity; for example, through the gradual local distribution of material properties to meet structural or environmental performance criteria. Robotic in situ processes also hold the potential to allow efforts in logistics to be minimized; e.g., no need for an off-site factory or intermediate stops for material transport. Thus, they could fundamentally change conventional construction site workflows, as well as the architectural language inherent to them.

While these architectural potentials highly motivate the expansion of the digital design-to-fabrication process chain to building sites, robotic in situ fabrication is still largely a potentiality as there are fundamental technological challenges - recently referred to as "in situ fabrication challenges" [23] - that have hindered its comprehensive implementation to date. One major challenge concerns the building scale. While robotic prefabrication relies on placing and fabricating building components within stationary work cells, robotic in situ

1 This means locally, on-site, or in place. 
fabrication requires the robot to be placed on site for it to reach and fabricate building structures at their final location. Another major challenge concerns the building site environment. While many well-established processes in robotic prefabrication rely on the complete knowledge and constant conditions of a structured factory environment, robotic processes on construction sites lack such certainty. Building sites are poorly structured and they also continuously change with the construction progress.

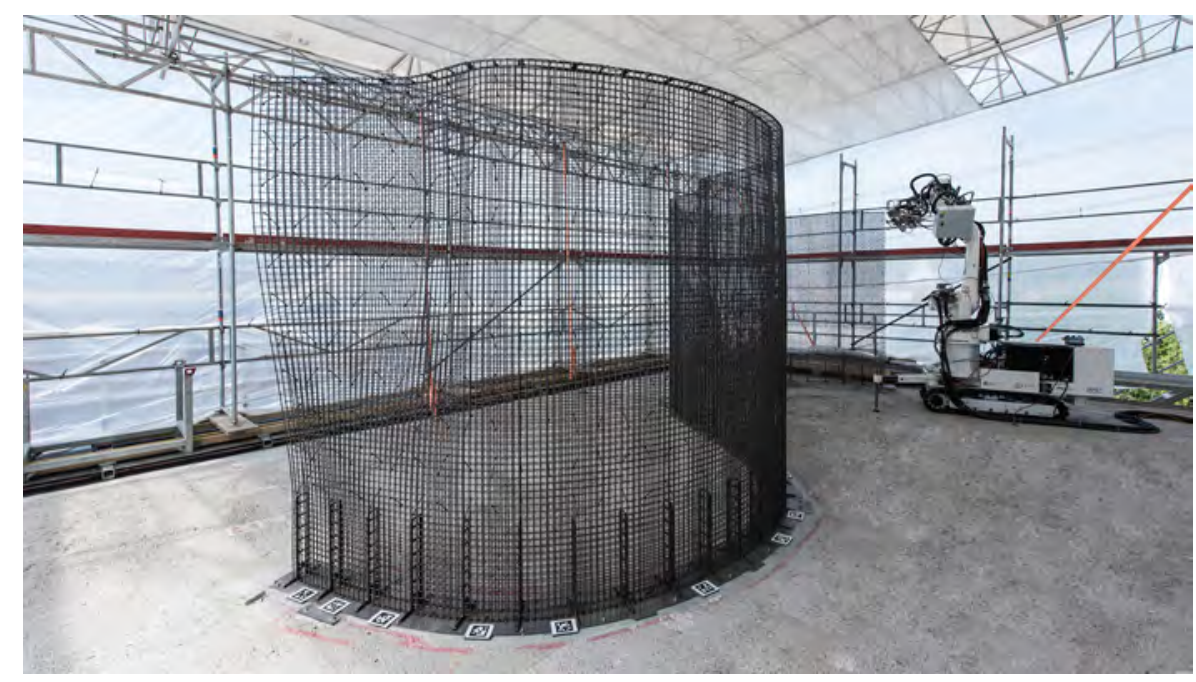

Fig. 2 The In situ Fabricator (IF) and Mesh Mould: The mobile construction robot IF is fabricating the last layers of the steel rebar mesh for the bespoke reinforced concrete wall on the DFAB HOUSE construction site, situated at the NEST building of Empa, Dübendorf, Switzerland.

The research presented in this paper addresses both of these challenges. First, to tackle the challenge of the architectural scale, this research explores mobile robotics to fabricate large-scale structures that exceed a robot's static workspace on a building site. For this purpose, a novel robotic construction system for reinforced concrete structures, which is designated as Mesh Mould (see [30] for details on the fabrication technology), was applied and customized. This construction system consists of robotically fabricating a double-sided hollow steel rebar mesh in a continuous layer-wise fashion using the mobile construction robot named the In situ Fabricator (IF, see [27] for technical details on the robot). Later, this hollow mesh effectively acts both as formwork for filling it with fresh concrete and as reinforcement for the hardened concrete. The fabrication technology has specifically been selected to demonstrate the full potential of using mobile robots on site; that is, the production of geometrically differentiated, structurally informed, and monolithic structures in both a material- and labor-efficient way. Second, to respond to the challenges arising from various uncertainties related to robotic in situ processes - the building 
site environment, the mobile robotic system, and the material behavior - this research proposes a sensor-integrated adaptive fabrication strategy. The fabrication system has been validated in various physical studies culminating in a demonstrator at full architectural scale - namely the mobile robotic fabrication of a doubly-curved steel rebar mesh for a fully load-bearing building structure known as the Mesh Mould wall of the DFAB HOUSE at NEST [43, 5] (Fig. 2).

The remainder of this paper is organized as follows. Section 2 elaborates on the background and motivation of this research. It provides a brief overview and assessment of existing strategies for robotic in situ fabrication, and it outlines the areas of focus for the experimental section of this paper. Section 3 provides details on the materials and methods used for this research and the realization of the architectural demonstrator. Section 4 reports the results and validation of this research, and lastly, Section 5 summarizes the results and discusses possible directions for future research.

\section{Background and motivation}

2.1 Recent developments of robotic in situ fabrication systems

In recent years, a number of companies and research institutions have increasingly addressed the challenge of robotic in situ fabrication of large-scale building structures. As depicted in the taxonomy in Fig. 3, various robotic systems have evolved that aim to meet the requirements of manipulation and assembly tasks at an architectural scale. Still, they vary greatly in regard to payload capacity, reach, flexibility, and construction process.

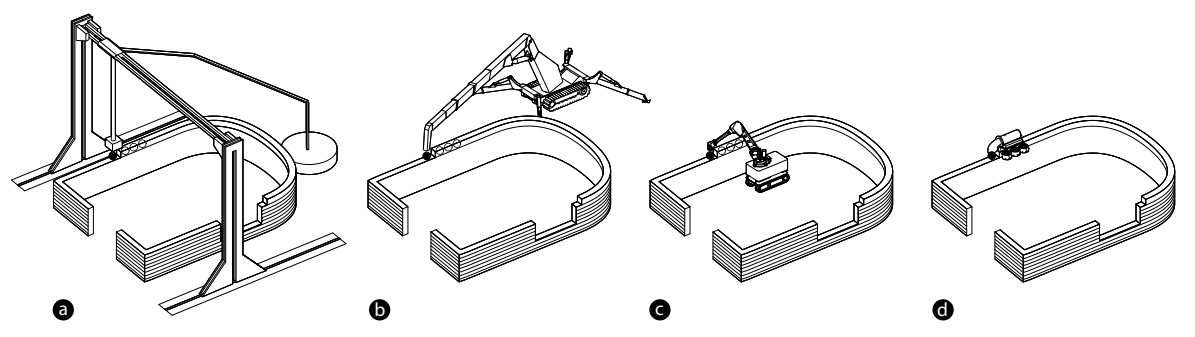

Fig. 3 Taxonomy of robotic systems for additive in situ fabrication. (a) Stationary systems with a wide-range static workspace (3D Printhuset BOD [1], ApisCor [2], BigDelta WASP [19]). (b) Mobile systems using an extended manipulator with a wide-range static workspace (Digital Construction Platform [34], Hadrian 105 [8]). (c) Mobile systems using a standard manipulator with a mid-range static workspace (DimRob [31], IF [27], CyBe RC 3Dp [4], SAM100 [3]). (d) Mobile systems without manipulator and a short-range static workspace (Minibuilders [11], TERMES [50], filament robots [12]).

Currently, the developed stationary systems $[1,2,19]$, as well as mobile systems with extended manipulators $[34,8]$ demonstrated the fabrication of ob- 
jects within their static workspace. One such example is the monolithic domelike extruded foam structure produced by the Digital Construction Platform, as depicted in Fig. 4(a). Having a wide-range workspace allows such systems to be stationary during fabrication and to thus rely on fixed mechanical links throughout their system. This facilitates their operation greatly in terms of planning and control. However, the size of the workspace eventually constrains the size of the object being fabricated.

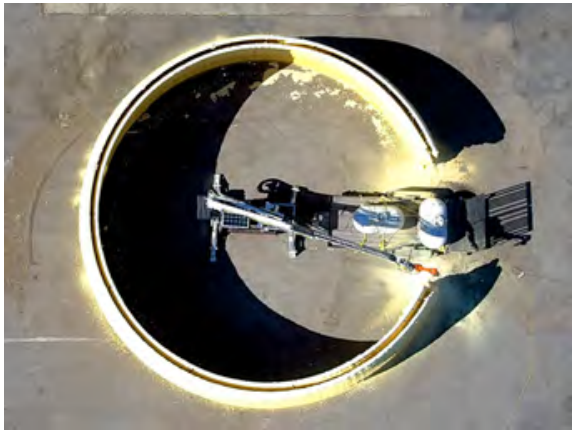

(a)

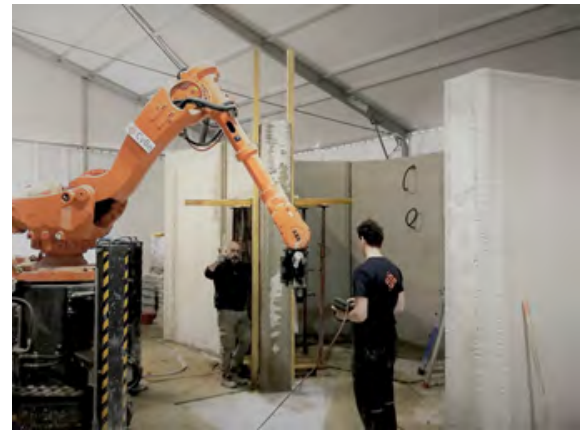

(b)

Fig. 4 (a) The Digital Construction Platform [34], a mobile dual-arm system with a widerange workspace developed at MIT, exhibited the in situ formwork fabrication of a largescale monolithic dome-like structure through foam extrusion within its static workspace (2017). (b) The mobile construction robot CyBe [4], an arm-based system with a mid-range workspace, has demonstrated the fabrication of a large-scale 3D printed concrete structure on site (2018). Due to the constraints of the additive processes, such as the curing of the concrete, the structure consists of 35 individual modules; each module complies with the static workspace limitations of the robot.

An approach to robotic in situ fabrication which overcomes this issue is to employ systems that are smaller in scale yet mobile. In mobile robotics, the system is, in principle, independent of the size or the shape of the object it produces. Despite the severe limitations in payload capacity for realistic construction scenarios, this concept has been convincingly demonstrated in projects involving mobile robots radically small in scale; examples include the Minibuilders project [11], and the TERMES project [50]. Mobile robots outperform stationary systems in relation to flexibility and dexterity, and they require only minimal effort to be set up on site [21]. However, mobile robots lack a rigid kinematic chain that links a robot's end effector to the ground. Due to the absence of such mechanical links in their systems during operation, the higher their mobility and flexibility is, the more their precise operation relies on advanced sensing and control solutions. This is complicated by the requirement to achieve absolute accuracy of large-scale constructions in a globally consistent coordinate system.

While current developments exhibit the potential application of mobile robotics to digital in situ fabrication, they also reveal several limitations. For 
example, recently published mobile robotic systems do not make use of stateof-the-art exteroceptive sensing and control solutions to enhance the autonomy, accuracy and robustness of such systems. They typically depend on external stationary referencing systems for localization and positioning the end effector with absolute accuracy on-site [3,13,52]. Visibility constraints and sensor range of such external installations eventually limit the flexibility and agility of a mobile system. Moreover, external measurement systems do not provide contextualized feedback beyond localization, and they cannot survey the built structure with respect to uncertain material processes. Lastly, constraints of a material system can cause the need to modularize building structures according to the static workspace limitations of the robot used; one such example is the robotic in situ process for $3 \mathrm{D}$ printed concrete components, depicted in Fig. 4(b).

\subsection{Uncertainties of building processes on site}

Processes in robotic prefabrication typically rely on complete knowledge provided by highly structured factory conditions. This relates to various factors such as dedicated work-cells, the absolute accuracy of physically bound stationary robotic systems, or the independence of outside weather situations. These conditions facilitate the planning and control of robotic procedures, and they ensure a high level of confidence in produced objects with respect to their digital design. Robotic in situ processes, however, are challenged by the lack of such complete knowledge, which is caused by a variety of external influences and the uncertainties of their immediate environment. For example, in comparison with their digital blueprints, building site environments often exhibit deviations and dimensional tolerances, particularly over large building spans. Such a discrepancy may occur if the as-planned digital model of a building site does not capture an accurate and detailed depiction of the actual as-built conditions; for instance, an entity may not be constructed exactly as the plan foresaw [46]. The exposure of building sites to the outer climate and temperature changes may cause deflections in constituent building elements, and hence, deviations from their digital models can occur. Furthermore, the building material can exhibit unpredictable behavior during the process of fabrication. ${ }^{2}$ Lastly, mobile robots lack a perfectly controlled system and can exhibit localization and positioning errors.

In robotic in situ fabrication, the predetermination of a digital model continually interferes with the unpredictability of the physical domain. In many cases, such prevalent uncertainties render a purely model-based approach as unstable, and create the need for a robot to continuously perceive its surroundings and obtain critical knowledge through exteroceptive sensing instead. This knowledge must then be used further within the process control in accordance

\footnotetext{
2 In prefabrication, robotic processes also have to cope with unpredictable material behavior. However, factors such as the prevalence of dirt and temperature changes increase the probability of materially induced uncertainties on-site.
} 
with defined performance criteria as the production progresses [25,40,44]. In this way, the control system can adapt the robot process to external stimuli and ensuring that the required fabrication accuracy is achieved.

\subsection{The in situ fabrication concept}

To fundamentally advance the technology and to develop robotic in situ construction methods within the field of architecture and digital fabrication, a synthetic and multi-disciplinary approach is required. Hence, this research is conducted in collaboration with researchers from several different fieldsnamely those of robotics, material science, structural engineering, and architecture.

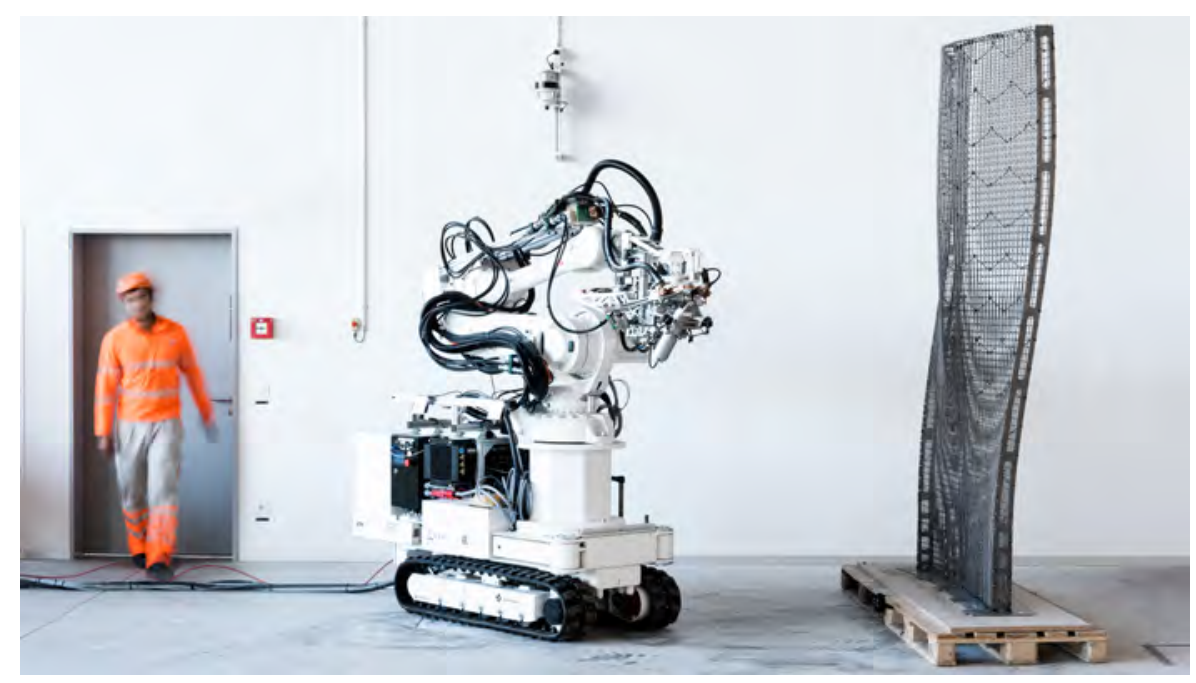

Fig. 5 The mobile robot IF is equipped with a custom-developed end effector to fabricate the steel rebar mesh for the Mesh Mould wall on site; in this image depicted with a full-scale mock-up of the mesh.

The payload capacities, reach, and flexibility of the mobile robotic system used for this research — named the In situ Fabricator (IF) — were designed and developed to fit the manipulation and assembly tasks at true architectural scale. The electrically powered arm-based system has a static reach of $3.2 \mathrm{~m}$ in height and $2.55 \mathrm{~m}$ in width, a payload capacity of $40 \mathrm{~kg}$, and a tracked hydraulically-driven mobile base. The prototype system IF described here is based on previous research within Gramazio Kohler Research and the NCCR Digital Fabrication that began in $2011[31,26,27]$. While prior experiments with the IF were performed in the lab, the aim of this research was to validate the use of the machinery in the context of a real-world construction scenario. To enhance the system's unobstructed mobility, its onboard sensing solutions 
were custom developed for the realization of the architectural demonstrator of this research; these solutions supported both the mobile robot's global localization on site and a local fabrication survey of the fabricated object. In addition, the mobile robotics approach also required advanced fabrication technology capable of exploiting its potential on a realistic architectural scale. The customization of the Mesh Mould fabrication technology [30,10] for this research enabled the mobile robot to continuously add material layer-by-layer while providing the structural integrity of the object during construction (Fig. 5). The robotic process was complemented by the subsequent manual filling of the produced structural formwork with concrete, which ultimately resulted in the fully load-bearing and monolithic architectural element. The research presented below also aims to reveal how the use of such distinct on-site robotic fabrication technology and its operational logic inform the architectural design process and create an architectural expression inherent to the very process.

\section{Materials and methods}

\subsection{Mesh Mould and In Situ Fabricator at DFAB HOUSE}

The robotic in situ process for a bespoke reinforced concrete wall using the IF by applying the Mesh Mould technology is featured as one of the innovation objects for the residential unit DFAB HOUSE in the Empa NEST building (Fig. 6) [6, 43].
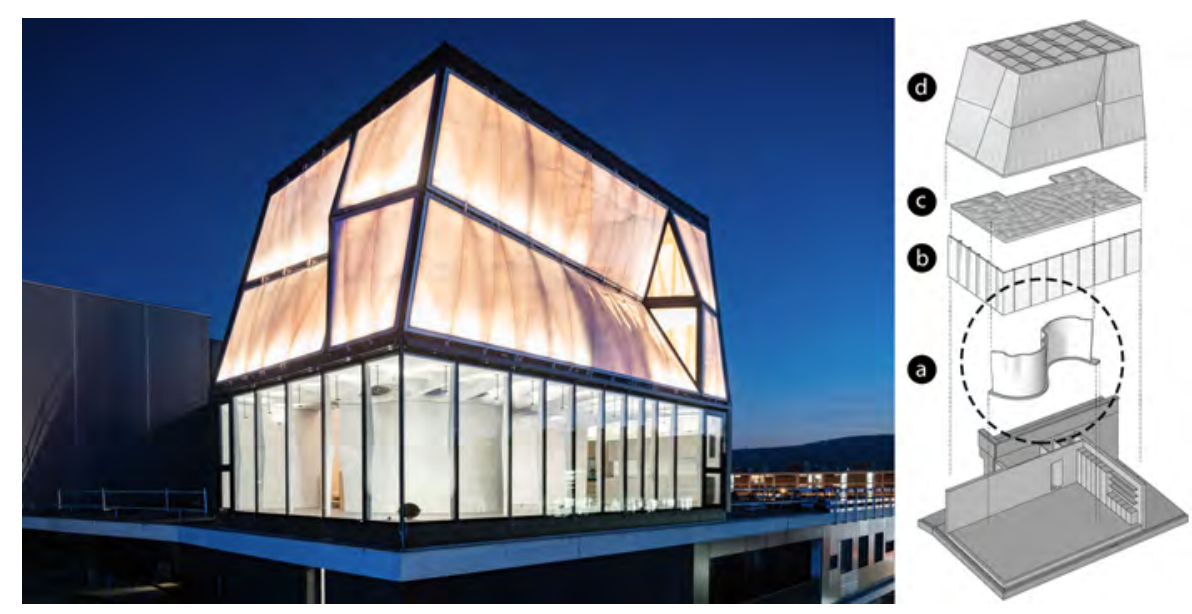

Fig. 6 Visualization of the DFAB HOUSE [5] of the Empa NEST building. The residential unit displays four innovation objects that aim to advance digital fabrication techniques in building construction: (a) In situ Fabricator and Mesh Mould [9], (b) Smart Dynamic Casting [14], (c) Smart Slab [15] and (d) Spatial Timber Assemblies [17]. 
The $12 \mathrm{~m}$-long, s-shaped, undulated, and monolithic Mesh Mould wall constitutes both a zoning element and the main load-bearing element on the ground floor of the unit. It supports a cantilevering pre-stressed concrete floor slab (Smart Slab [16]) topped with a two-story timber structure (Spatial Timber Assemblies [17]). In total, the wall carries $80 \mathrm{t}$ of vertical and lateral loads.

\subsection{Fabrication-aware computational design}

A process that is dependent on its combined sources of material, structural, and fabrication-related knowledge informed the design of the wall. Primarily, the design intended to increase the structural capacity of the wall through a differentiated geometry, thereby reducing the wall's overall thickness and material consumption. This was achieved by locally undulating and introducing a double curvature at specific locations along the wall's surface. Together with assessing its structural performance, fabrication-related constraints were continually considered along the respective design workflow (Fig. 7; see also [30] for details on the structural analysis of the Mesh Mould material system). This approach allowed the design space of feasible differentiated geometries related to the material system, structure, and fabrication to be explored. While the realization of such a complex geometry would be expensive and labor-intensive if built using traditional methods, e.g., by using formwork, this research aimed to demonstrate the efficiency of applying the in situ fabrication of the Mesh Mould technology instead.
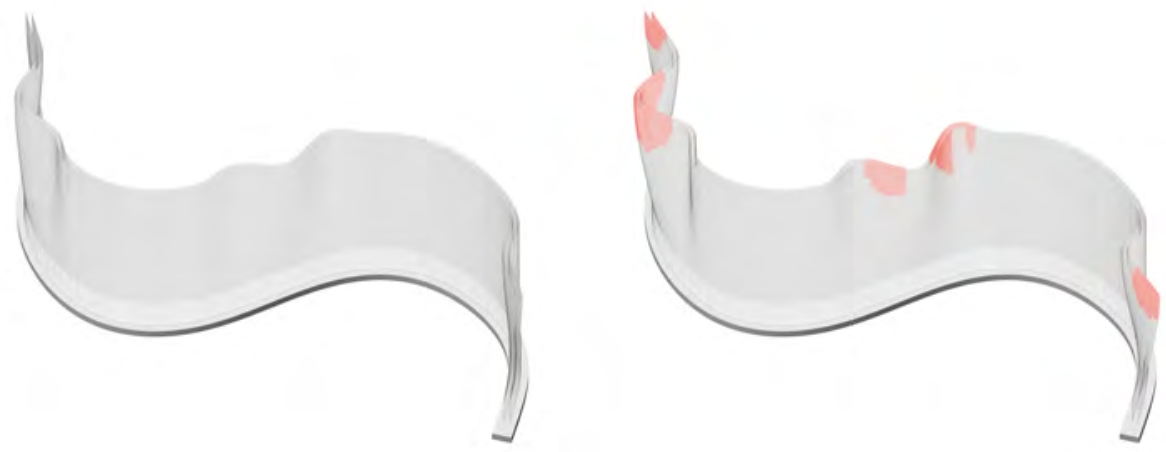

Fig. 7 Using the custom design tool for generating wall geometries, designers can study the structural effects of locally undulating various surface geometries. At the same time, the design tool informs the designer also about fabrication-related constraints. As illustrated by the example on the right, a high undulation amplitude results in exceeding fabrication constraints such as the limits for the layer height between rebar elements at certain regions of the mesh. These regions are marked in red accordingly.

To form a geometrically differentiated and stable reinforcement mesh, a custom-developed end effector integrated into the IF successively cross-wire 
welded layers of long, continuous, ribbed steel rebar $(6.0 \mathrm{~mm}$ in diameter $)$ with short, discrete elements of ribbed steel rebar $(4.5 \mathrm{~mm}$ in diameter). The layout of the steel rebar had to meet requirements derived from structural considerations and constraints imposed by the mesh-filling process (see $[37,30]$ for further details), as well as mobile fabrication-related considerations. Hence, the layers of the continuous steel rebar elements were arranged in the vertical direction as opposed to the horizontal direction of layers used in conventional $3 \mathrm{D}$ printing processes. This had the advantage of creating continuous strands of rebar complying with the predominant vertical direction of the forces acting on the wall. More importantly to the mobile robotic in situ process, it allowed the mesh to expand in the direction of the robot's movement. This fabrication logic enabled the IF to construct the full $2.8 \mathrm{~m}$ height of the wall at once, and to complete the $12.0 \mathrm{~m}$-long monolithic wall through continually adding vertical layers of rebar elements and by relocating only eight times (Fig. 8).

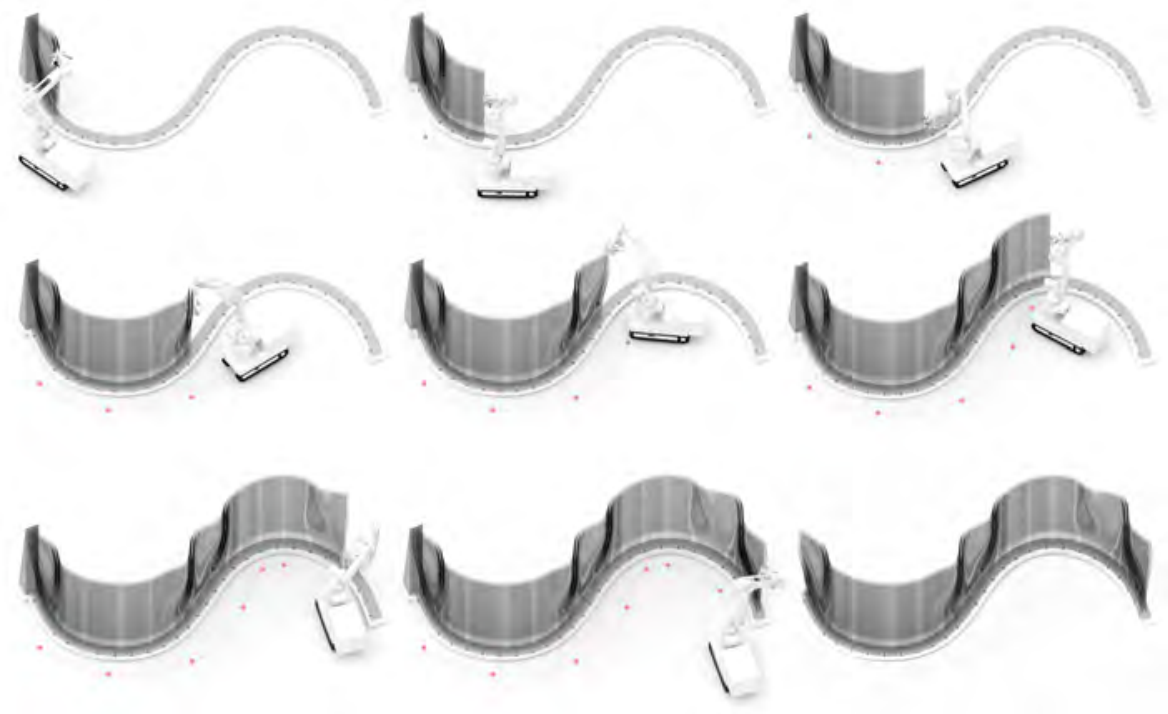

Fig. 8 Visualization of the mobile fabrication sequence: The mesh of the wall is fabricated in vertical layers to its full $2.8 \mathrm{~m}$ height. The vertical arrangement of the layers allows the structure to be expanded horizontally along the mobile robot's path. To complete the $12.0 \mathrm{~m}$-long mesh construction, the IF has to be relocated only eight times.

3.3 Sensor-integrated adaptive fabrication strategy

With respect to the high accuracy requirements for the mesh fabrication - both in relation to the robustness of the process (e.g., avoiding collisions between 


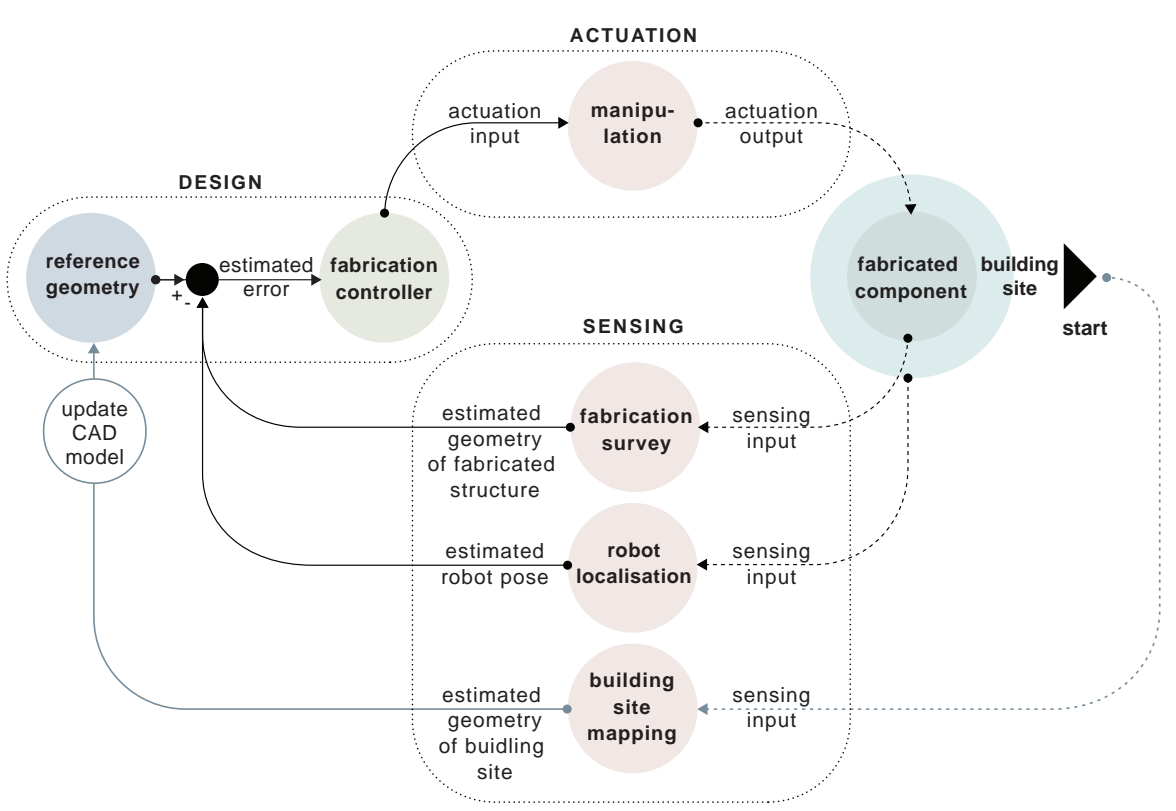

Fig. 9 Diagram of the sensor-integrated adaptive fabrication strategy: The geometric-based closed-loop control allows the robotic system to deal with uncertainties related to the (1) building site, (2) the robot localization, and (3) the material behavior. The diagram should also show the close entanglement of design, actuation, and sensing in robotic in situ fabrication.

the end effector and the mesh) and the resulting as-built accuracy of the constructed object - this research has established and subsequently validated a sensor-integrated adaptive fabrication strategy (Fig. 9). This adaptive fabrication strategy involves integrating the following steps into a geometric-based closed-loop control method. A priori to fabrication, a robot must sense and capture the building site environment and create a 3D representation of itthis is referred to as building site mapping. In one calibration step, the globally defined geometric representation of the building site, including the reference geometry for fabrication, must be aligned with the obtained map. In the case of deviations between the as-planned reference geometry to the obtained as-built map, the reference geometry must be adapted to the true dimensions found onsite; such an alignment procedure has also been depicted in previous research in [26]. During fabrication, the fabrication controller continually monitors the difference between the desired dimensions (i.e., the reference geometry) and the estimated dimensions (i.e., the sensor feedback) of the fabricated object with sensory information coming from multiple sources; typically, the sources are the fused estimations of both the global robot localization and the local measurements of the fabricated object. The fabrication controller then iteratively generates control actions to achieve the desired performance criteria. Through these control actions, robotic manipulation procedures can be incre- 
mentally adjusted to attain the globally defined reference geometry, and the robot can cope with the uncertainties induced by multiple sources on site.

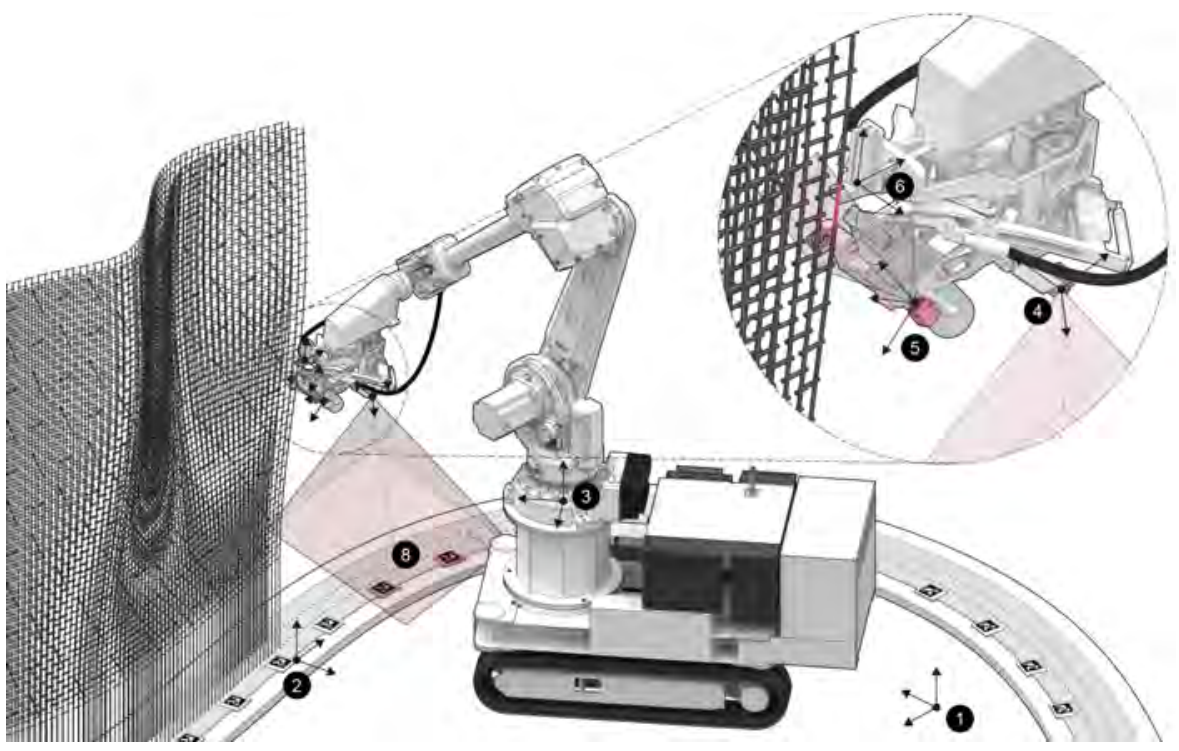

Fig. 10 Illustration of the IF's complementary vision-based sensing solution with one camera at the end effector that is used for the robot's global localization, and two more cameras for the in-process fabrication survey of the rebar mesh. The image also displays the various coordinate frames (cf) used in the sensing system: (1) world cf, (2) fiducial marker cf, (3) robot cf, (4) localization camera cf, (5) fabrication survey camera cf, and (6) end effector cf [38].

To implement the adaptive fabrication strategy for the mesh fabrication process with the IF, an integrated onboard vision-based sensing solution has been developed that consists of two complementary parts (Fig. 10, and see [38] for technical details on the implementation). The first part concerned the building site mapping and the robot's global localization on the job site; this was achieved by the IF firstly mapping and subsequently registering AprilTag fiducial markers mounted along the wall's foundation area. The second part concerned the local fabrication survey. This was achieved by the IF iteratively registering the contour of the rebar elements within layer's of the mesh. The subsequent feedback of the sensing data into the fabrication control allowed the robot to compensate locally for two main sources of error-that is, firstly for deflections of the mesh (e.g., due to inner forces caused by the steel rebar's elastic deformation or by the welding heat), and secondly for estimation inaccuracies in the robot's global localization (Fig. 11). 


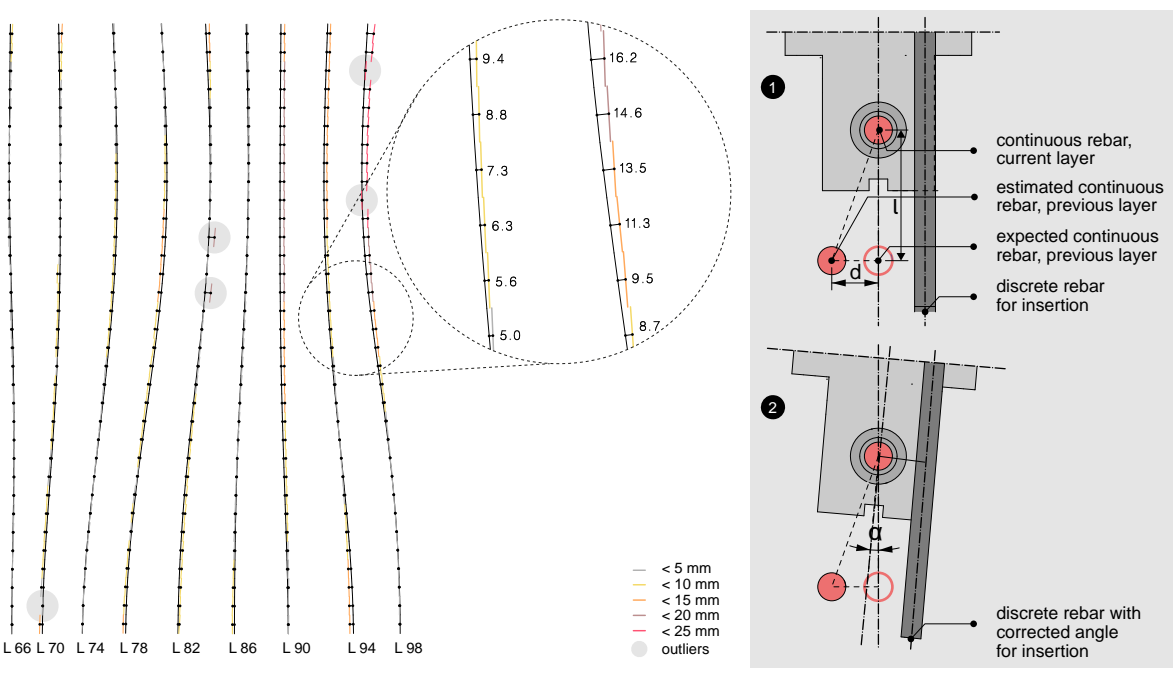

Fig. 11 Sequences of welding nodes forming the rebar mesh contour at full layer height, depicting the comparison of the estimated location obtained from the onboard vision system to the expected location in the CAD model. A color code indicates the amount of deviation, i.e., distances from the midpoint of the estimated line segments to the midpoint of the expected line segments. Outliers are marked with a gray circle (left). According to the obtained error $d$, the fabrication controller continually adapts the welding angle $\alpha$ for each welding node towards the desired reference geometry (right).

\section{Results and validation}

The 2.8-m high doubly curved steel rebar mesh consisting of 339 layers with more than 22000 welding nodes was successfully constructed from eight different robot locations in approximately 125 hours of production time at the DFAB HOUSE construction site (Fig. 12; see also the movie documentation of the process in [9]).

The net cycle time measured for each welding node was around $5 \mathrm{~s}$ per node, but it was observed that the gross time observed was around $20 \mathrm{~s}$ per node. The gross cycle time included several additional tasks, such as the visual survey of the mesh contour in between layers of the mesh, several manual interventions such as material feeding, the insertion of a steel support elements (i.e., foundation plates, top plates, cross connections), and the robot relocation procedures. At this fabrication speed, on average 16 to 24 layers could be fabricated each day and the robot was relocated every second day (see also Table 1 for production figures, and an elaborate productivity assessment for the wall construction in [45]).

Relocating the robot took approximately 1 hour, and it included the remote controlled driving of the robot platform, the manual relocation of the robot's periphery and the automated localization procedure using the onboard vision system. The localization method was refined and calibrated during the first five robot relocations procedures. This calibration was achieved by estimating 


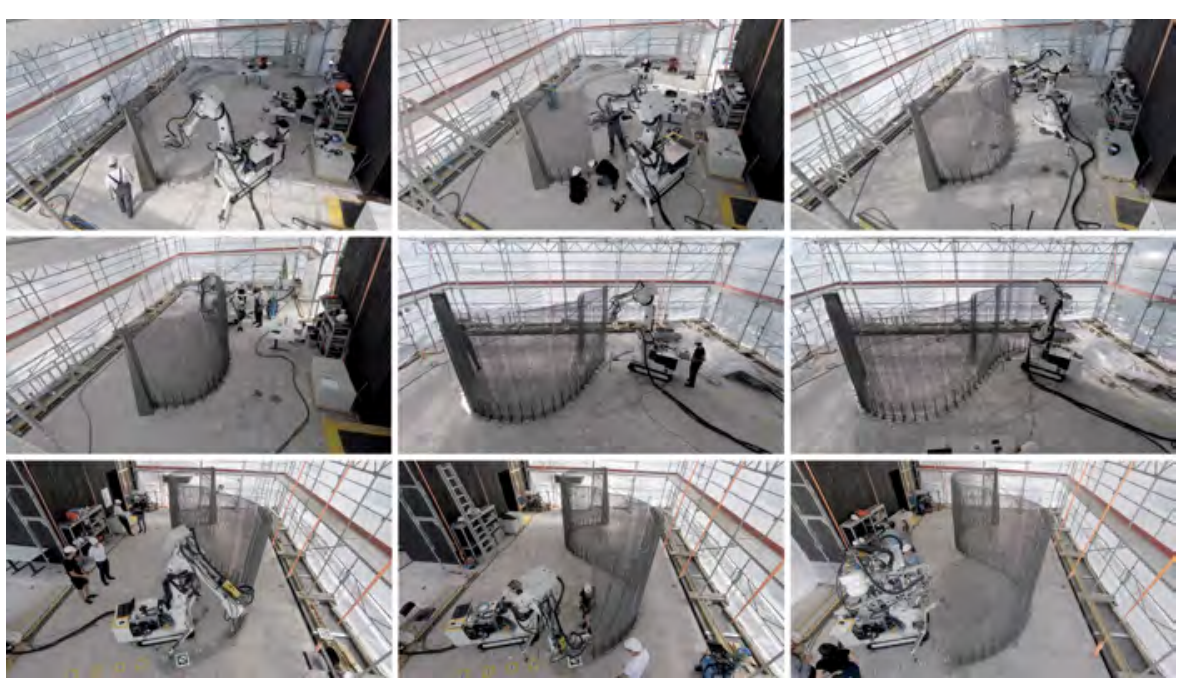

Fig. 12 Timelapse snaphots of the IF fabricating the rebar mesh on the DFAB HOUSE building site from subsequent locations. The whole fabrication process is also documented as a video and can be viewed online at [9].

the robot pose manually with a steel probe tip on the end effector and comparing this manual estimation with the automated estimation. It was observed that the best estimation was achieved by measuring the fiducial markers from the same distance and angle from which their map was created (from approximately a $1-\mathrm{m}$ distance and an angle between $20^{\circ}$ and $30^{\circ}$ ). For the last three robot relocation procedures only the visual estimation was used, and no additional manual measuring for comparing the results was necessary. Finally, the local error compensation enabled by the adaptive fabrication strategy allowed the mesh to be fabricated without collision and as a freestanding structure.

The accuracy of the finished mesh fabrication was validated by taking ground truth measurements using a Faro laser scanner. The validation proved the performance of the system to be consistent and predictable, thus confirming that the system achieves construction industry standard benchmarks (Fig. 13).

Following the robotic process, the steel mesh was manually reinforced with additional rebar elements to enhance the structural capacity at the open endings of the wall and the higher doubly curved regions. To avoid major deformations during the subsequent concreting process, the mesh was also temporarily diagonally braced using steel posts at six locations along the wall (Fig. 14(a)). Finally, the mesh was filled with a MonoTop-412 N cement mix and covered with a layer of Sika MonoTop-352 N shotcrete for the surface finish, resulting in a fully load-bearing and monolithic building element (Fig. 14(b)). 


\begin{tabular}{|l|l|}
\hline \multicolumn{2}{|c|}{ Dimension, speed, accuracy, and robustness figures of the fabrication process } \\
\hline Wall height / length top / length bottom & $2.8 \mathrm{~m} / 11.8 \mathrm{~m} / 11.4 \mathrm{~m}$ \\
\hline Number of vertical layers & 339 \\
\hline Minimum / maximum distance between vertical layers & $31 \mathrm{~mm} / 41 \mathrm{~mm}$ \\
\hline Number of welding nodes of one double-sided vertical layer & $66 / 68$ \\
\hline Number of welding nodes & 22374 \\
\hline Number of robot relocations & 8 \\
\hline $\begin{array}{l}\text { Time required for robot relocations incl. manual assistance of } \\
\text { remote controlled driving and moving the robot's periphery }\end{array}$ & $1 \mathrm{~h}$ \\
\hline Visual survey time of one welding node / one layer & $4.3 \mathrm{sec} / 2.5 \mathrm{~min}$ \\
\hline Net fabrication time of one welding node / one layer & $5 \mathrm{sec} / 5.5 \mathrm{~min}$ \\
\hline $\begin{array}{l}\text { Gross fabrication time of one welding node / one layer (incl. } \\
\text { visual fabrication survey, manual auxiliary processes) }\end{array}$ & $20 \mathrm{sec} / 22 \mathrm{~min}$ \\
\hline $\begin{array}{l}\text { Percentage of time required for automated procedures vs. } \\
\text { manual auxiliary procedures }\end{array}$ & $33.3 \%$ vs. $66.6 \%$ \\
\hline Layers fabricated daily & $16-24$ layers \\
\hline Total gross fabrication time & $125 \mathrm{~h}$ \\
\hline Estimated global accuracy of the end effector positioning & $\pm 15 \mathrm{~mm}$ \\
\hline Estimated local accuracy of the mesh contour survey & $\pm 5 \mathrm{~mm}$ \\
\hline Percentage of outliers in the mesh contour detection & $1.6 \%$ \\
\hline
\end{tabular}

Table 1 Figures on the speed, accuracy, and robustness of the Mesh Mould rebar mesh fabrication using the mobile robot IF.

$\geq 20.0 \mathrm{~mm}(2.1 \%)$

$\geq 16.7<20 \mathrm{~mm}(2.5 \%)$

$\geq 13.3<16.7 \mathrm{~mm}(7.8 \%)$

$\geq 10.0<13.3 \mathrm{~mm}(20.4 \%)$

$<10.0 \mathrm{~mm}$ (67.1\%)

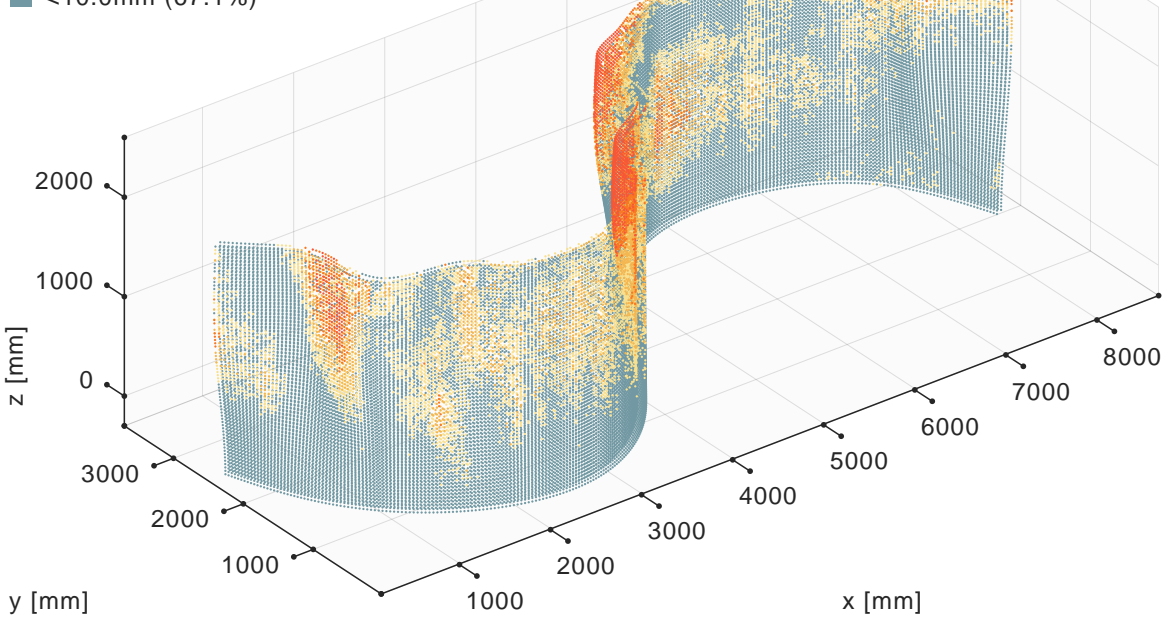

Fig. 13 Error plot computation based on ground truth measurements of the fabricated mesh: The colors represent regions that fall within different error ranges. and $98 \%$ of the steel rebar mesh stayed well below $20 \mathrm{~mm}$ of tolerances. The severest mesh deflections appear in the regions with a higher curvature and towards the top of the wall [38]. 


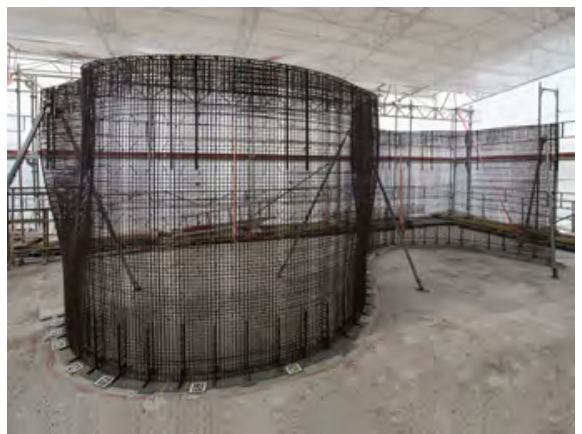

(a)

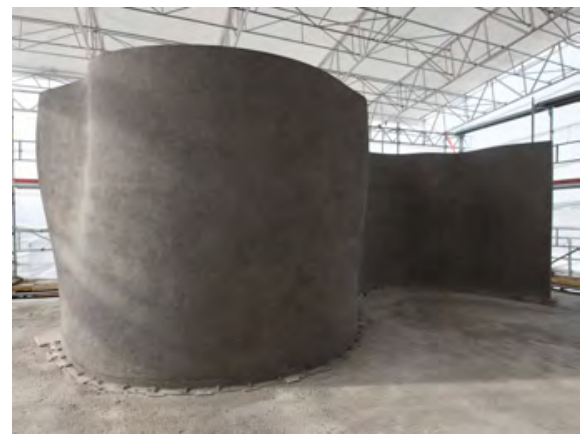

(b)

Fig. 14 (a) For structural reasons, the steel rebar mesh required to be manually reinforced at the open endings of the wall and the higher doubly curved areas. (b) The robotically fabricated mesh was then manually filled with a cement mixture and surface finished with a layer of shotcrete, resulting in a fully load-bearing and monolithic building element $[30,10]$.
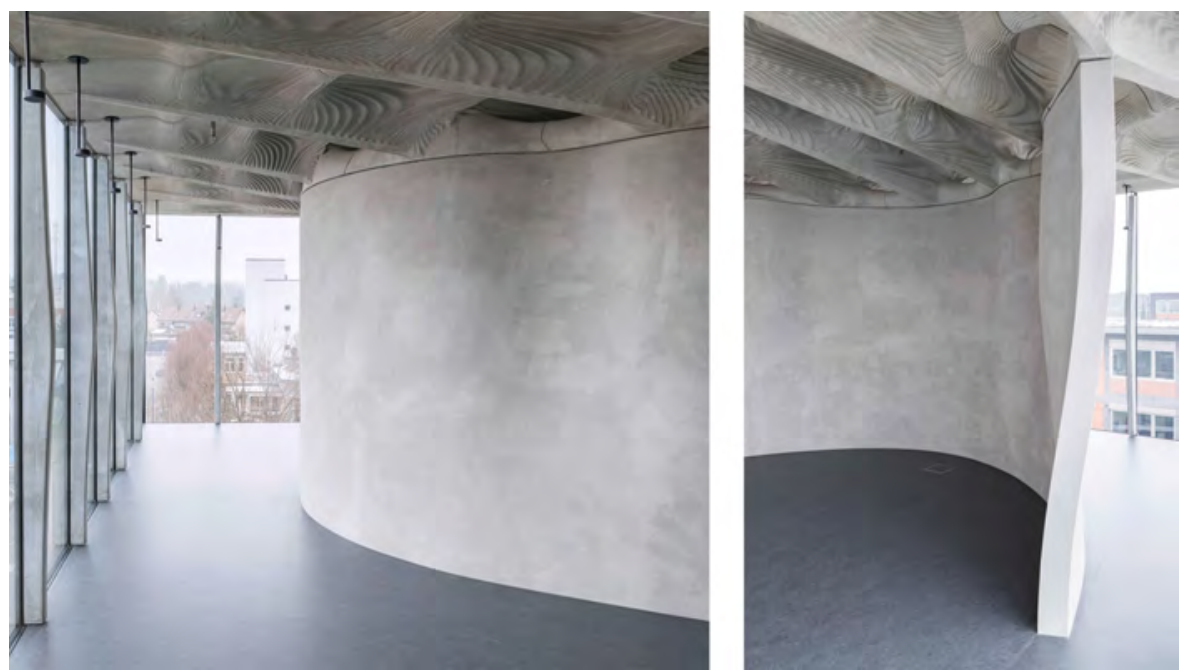

Fig. 15 The finished doubly-curved slender Mesh Mould wall at the DFAB HOUSE supporting the cantilevering pre-stressed concrete Smart Slab with a wall thickness of $12.5 \mathrm{~cm}[16,10]$. 


\section{Discussion}

\subsection{Conclusion}

This research developed and experimentally validated methods and strategies that addressed two major robotic in situ fabrication challenges: the first one concerns the large scale of building construction, and the second one concerns the accuracy required for fabrication. The following conclusions can be drawn from the strategies applied:

\section{Architectural scale}

The presented robotic in situ process demonstrated the realization of a largescale and monolithic building structure through mobile fabrication, exceeding the mobile robot's static workspace in the horizontal plane. The size of the produced object is still constrained by the robot's reach in height. However, the process nevertheless ushers fabrication towards the robotic production of building structures that are designed to be free of components and with fewer limitations which are usually imposed by transport, final positioning, and assembly.

\section{Accuracy}

To make the mobile robotic process on a building site possible - that is, independent of structured factory conditions - the research presented an adaptive fabrication strategy in which the robot captured critical knowledge using its onboard sensing systems by 1) mapping the geometry of the building site, 2) localizing the robot origin within it, and 3) surveying the ongoing fabrication process, and the fabrication controller continually integrated this knowledge for the generation of the robot tasks.

With respect to this strategy, this experiment attained a number of findings and achievements. Firstly, the mapping of the fiducial markers placed on the construction site allowed the robot to align and adapt the CAD model of them to the obtained map. Secondly, the robot localization in reference to the markers enabled the robot to move on the job site and to position its end effector in a globally consistent world frame. Finally, the fabrication survey facilitated the robot to constantly monitor whether its actions succeeded reliably. This allowed the robot to locally contend with uncertain factors -including both the unpredictable material behavior and noise in the localization estimationsand eventually it could fabricate a structure that accurately adhered to the globally defined reference geometry. 


\subsection{Future research}

\section{Sensor integration and open-ended form-generation}

In contemporary design culture, it is hardly ever questioned, that design and form-generation processes are preceding materialization processes [42]. Adaptive fabrication, however, challenges the notion of a unidirectional information flow from the design to the fabricated object $[25,44]$. In contrast to the material being merely a passive receiver of a predetermined form, the introduction of feedback loops allows the material to play an active role in the physical formation process $[33,48]$.

In the presented research, the feedback of the geometric sensing data obtained from three different sources (i.e., the robot map, the localization estimation, and the fabrication survey) allowed the fabrication control loop to be closed in a globally consistent coordinate system. It enabled the robot to locally compensate for prevalent uncertainties on the building site and fabricate the building component in reference to the predefined design and performance criteria. As a result, the built structure deviated locally from the dimensions of the predefined digital reference model; in particular, the local material formations differed in appearance from the model. From the global perspective, however, the digital model and the built structure were both consistent; in this case, deviations and errors did not accumulate. In extension to this approach, future research could explore an open-ended design approach to fabrication, in which the form-generation processes are informed by material properties and behavior during the form's materialization not only at the level of local material formations but also on the level of the global form. Integrating design with sensor-integrated fabrication control could unlock the generative potential of such a process $[49,41,35]$. Subsequently, future research could explore the implications of such an approach on the architectural design and form-generation processes at large.

\section{Dynamic mobile fabrication}

With respect to future research, the mobility, agility, and associated dynamics of a robotic system for in situ fabrication require substantial improvement. The presented in situ process demonstrated fabrication and relocation procedures in successive discrete steps. However, having a mobile robot capable of dynamic manipulation would allow an exploration of the potentials for performing these procedures to occur simultaneously. Such an increased mobility and agility would expand the application space to continuous construction processes. For example, this would make concrete 3D printing free of modularization possible, or in the specific case of the Mesh Mould technology, it would allow for continuous robotic concrete extrusion processes directly onto a steel rebar mesh. 
Local differentiation

Robotic fabrication supports the distribution of properties and geometric differentiation in the materialization process of an architectural object in various scales. The presented process demonstrated the realization of locally undulated - and thus differentiated - surface geometry. However, this geometry was fabricated through homogeneous material addition (i.e., by evenly distributed steel rebar cells, in width and height), with a constant wall thickness and a constant concrete mixture for filling. Due to structural requirements, the mesh with the evenly distributed steel rebar then required manual post reinforcement at the open endings of the wall and at regions with a higher double curvature. Future research could integrate such local variations well into the robotic fabrication process. Moreover, future research could also explore a higher degree of material heterogeneity in accordance with loading conditions or environmental demands by varying the distribution of properties on various levels such as material density, porosity, and geometric articulation.

\section{Sustainability}

The shrinking resources of construction material, the still growing need for building construction, and the necessity for high-quality working conditions and well-built environments are some of the fundamental challenges facing our world today. There can be no intellectual, conceptual, or societal separation between the architecture that we produce and the techniques with which we produce it. As digital technology is essential to the discipline and practice of architecture, the ability to shape and innovate with technology to tackle these challenges is urgent and greatly important. As an alternative to robotic prefabrication, robotic on-site fabrication has the potential to minimize the logistical efforts and the energy consumption of building construction, as already indicated by the assessment of the Mesh Mould wall case study by [40]. Moreover, the geometric differentiation of building structures enabled by advanced digital design and fabrication tools, allows to enhance the material and structural performance, and consequently, the overall material consumption to be minimized. Hence, finding a common path towards a sustainable future makes architectural production as much a technological endeavor as a design project.

Acknowledgements This research was supported by the Swiss National Science Foundation through the NCCR Digital Fabrication (NCCR Digital Fabrication Agreement 51NF40141853) and a Professorship Award to Jonas Buchli (Agreement PP00P2-138920). The authors would like to thank a number of people from ETH Zurich who were, directly and indirectly, involved in the research, including Konrad Graser and Marco Baur (project architects DFAB HOUSE), Michael Lyrenmann, Philippe Fleischmann, and Andreas Reusser (head of technicians and technicians of the Robotic Fabrication Laboratory), Dr. Nitish Kumar, Julio Alonso Lopez, and Lukas Stadelmann (Agile and Dexterous Robotics Lab, Prof. Dr. Jonas Buchli), Dr. Timothy Wangler, Lex Reiter, and Heinz Richner (Physical Chemistry of Building Materials group, Prof. Dr. Robert J. Flatt), Dr. Jaime Mata-Falcón 
(Institute of Concrete Structures and Bridge Design, Prof. Dr. Walter Kaufmann), and Dr. Andrew Liew (Block Research Group, Prof. Dr. Philippe Block).

\section{References}

1. 3D Printhuset - The BOD. URL https://3dprinthuset.dk/the-bod/

2. Apis Cor Construction. URL http://apis-cor.com/en/

3. Construction Robotics. URL http://www.construction-robotics.com/

4. CyBe Construction. URL https://cybe.eu/3d-concrete-printers/

5. DFAB HOUSE. URL http://dfabhouse.ch

6. Empa NEST. URL https://www.empa.ch/web/nest

7. ERNE Bau AG - Gantry Robot. URL https://www.erne.net/de/leistungen/technologien/

8. FastBrick Robotics. URL http://www.fbr.com.au/

9. In situ Fabricator and Mesh Mould. URL https://www.youtube.com/watch?v=ZeLEeY8yK2Y

10. Mesh Mould Wall of DFAB HOUSE. URL http://dfabhouse.ch/mesh ${ }_{m}$ ould/

11. Minibuilders. URL http://robots.iaac.net/

12. Mobile Robotic Fabrication System for Filament Structures. URL http://icd.unistuttgart.de/?p=15699

13. Nlink Drilling Robot. URL https://www.nlink.no/

14. Smart Dynamic Casting of DFAB HOUSE. URL http://dfabhouse.ch/smart-dynamiccasting/

15. Smart Slab of DFAB HOUSE. URL http://dfabhouse.ch/smart-slab/

16. Smart Slab of DFAB HOUSE. URL http://dbt.arch.ethz.ch/project/smart-slab/

17. Spatial Timber Assemblies of DFAB HOUSE. URL http://dfabhouse.ch/de/spatial imber $_{\text {a ssemblies / }}$

18. Taichung opera house. URL https://www.dezeen.com/2016/10/01/this-week-toyo-itotaichung-opera-house-zaha-hadid-amanda-levete-apple-riba-elon-musk/

19. WASP BigDelta. URL http://www.wasproject.it/w/en/tag/bigdelta-en/

20. Adel, A., Thoma, A., Helmreich, M., Gramazio, F., Kohler, M.: Design of Robotically Fabricated Timber Frame Structures. 38th Annual Conference of the Association for Computer Aided Design in Architecture (ACADIA 2018), Mexico City, Mexico, October 18-20, 2018 p. 403. URL http://hdl.handle.net/20.500.11850/314075

21. Ardiny, H., Witwicki, S., Mondada, F.: Construction automation with autonomous mobile robots: A review. International Conference on Robotics and Mechatronics, ICROM 2015 pp. 418-424 (2015). DOI 10.1109/ICRoM.2015.7367821

22. Brell-Çokcan, S., Braumann, J. (eds.): Rob/Arch 2012: Robotic Fabrication in Architecture, Art and Design (2013). DOI 10.1007/978-3-7091-1465-0. URL http://link.springer.com/10.1007/978-3-7091-1465-0

23. Buchli, J., Lussi, M., Giftthaler, M., Dörfler, K., Sandy, T., Hack, N., Kumar, N.: Digital in situ fabrication - Challenges and opportunities for robotic in situ fabrication in architecture, construction, and beyond. Cement and Concrete Research (2018)

24. Callicott, N.: The tacit component and the numerical model: Representation in computer-aided manufacture and architecture. Journal of Architecture 8(2), 191-202 (2003). DOI 10.1080/1360236032000106025

25. Dörfler, K., Rist, F., Rust, R.: Interlacing - An Experimental Approach of Integrating Digital and Physical Design Methods. In: S. Brell-Çokcan, J. Braumann (eds.) Rob-Arch 2012 - Robotic Fabrication in Architecture, Art and Design, pp. 82-91. Springer-Verlag Wien, Vienna (2012). DOI 10.1007/978-3-7091-1465-0_7

26. Dörfler, K., Sandy, T., Giftthaler, M., Gramazio, F., Kohler, M., Buchli, J.: Mobile Robotic Brickwork: Automation of a Discrete Robotic Fabrication Process Using an Autonomous Mobile Robot. In: Robotic Fabrication in Architecture, Art and Design 2016, pp. 204-2017 (2016). DOI 10.1007/978-3-319-04663-1

27. Giftthaler, M., Sandy, T., Dörfler, K., Brooks, I., Buckingham, M., Rey, G., Kohler, M., Gramazio, F., Buchli, J.: Mobile Robotic Fabrication at 1:1 scale: the In situ Fabricator. Construction Robotics 1(1-4), 3-14 (2017). DOI 10.1007/s41693-017-0003-5. URL http://arxiv.org/abs/1701.03573 
28. Gramazio, F., Kohler, M.: Digital Materiality in Architecture. Lars Müller Publishers, Baden (2008)

29. Gramazio, F., Kohler, M., Willmann, J.: The Robotic Touch - How Robots Change Architecture. Park Books (2013)

30. Hack, N., Wangler, T., Mata-Falcon, J., Dörfler, K., Kumar, N., Walzer, A.N., Graser, K., Reiter, L., Richner, H., Buchli, J., Kaufmann, W., Flatt, R.J., Gramazio, F., Kohler, M.: Mesh Mould: An On Site, Robotically Fabricated, Functional Formwork. In: Proceedings of the Second Concrete Innovation Conference (2nd CIC) (2017)

31. Helm, V., Ercan, S., Gramazio, F., Kohler, M.: Mobile robotic fabrication on construction sites: DimRob. In: 2012 IEEE/RSJ International Conference on Intelligent Robots and Systems, pp. 4335-4341. Vilamoura, Algarve, Portugal (2012)

32. Ingold, T.: The textility of making. Cambridge Journal of Economics 34(March), 91-102 (2010). DOI 10.1093/cje/bep042

33. Johns, R.L.: Augmented Materiality: Modelling with Material Indeterminacy. In: F. Gramazio, M. Kohler, S. Langenberg (eds.) Fabricate: Negotiating Design and Making. gta-Verlag (2014)

34. Keating, S.J., Leland, J.C., Cai, L., Oxman, N.: Toward site-specific and self-sufficient robotic fabrication on architectural scales. Science Robotics 2(5), 15 (2017). DOI 10.1126/scirobotics.aam8986

35. Knight, T.: Craft, Performance, and Grammars. 2nd International Workshop on Cultural DNA (2017)

36. Krechting, A.: Prefabrication in the Brick Industry. In: 13th International Brick and Block Masonry Conference, pp. 1-10 (2004)

37. Kumar, N., Hack, N., Dörfler, K., Walzer, A., Rey, G., Gramazio, F., Kohler, M. Buchli, J.: Design, Development and Experimental Assessment of a Robotic Endeffector for Non-standard Concrete Applications. In: IEEE International Conference on Robotics and Automation (ICRA 2017), pp. 1707-1713. IEEE International Conference on Robotics and Automation (2017)

38. Lussi, M., Sandy, T., Dörfler, K., Hack, N., Gramazio, F., Kohler, M., Buchli, J.: Accurate and Adaptive In situ Fabrication of an Undulated Wall using an On-Board Visual Sensing System. In: Proceedings - IEEE International Conference on Robotics and Automation (ICRA 2018) (2018)

39. McKinsey Global Institute: Reinventing Construction: A Route To Higher Productivity. McKinsey Quarterly (February), 168 (2017). URL http://www.mckinsey.com/industries/capital-projects-and-infrastructure/ourinsights/reinventing-construction-through-a-productivity-revolution

40. Menges, A.: Material Computation. Architectural Design 82(2), 256-265 (2012)

41. Menges, A.: The new cyber-physical making in architecture: Computational construction. Architectural Design 85(5), 28-33 (2015). DOI 10.1002/ad.1950

42. Oxman, N., Mitchell, W.J., Arts, M., Supervisor, T., Beinart, J.: Material-based Design Computation (2010). URL http://hdl.handle.net/1721.1/59192

43. Richner, P., Heer, P., Largo, R., Marchesi, E., Zimmermann, M.: NEST - A platform for the acceleration of innovation in buildings 69, 1-8 (2017). DOI 10.3989/id.55380

44. Rust, R., Jenny, D., Gramazio, F., Kohler, M.T., Rust, R., Jenny, D., Gramazio, F., Kohler, M.: Spatial Wire Cutting - Cooperative robotic cutting of non-ruled surface geometries for bespoke building components pp. 529-538 (2016)

45. Soto, B.G.D., Agustí-juan, I., Hunhevicz, J., Habert, G., Adey, B.: The potential of digital fabrication to improve productivity in construction: cost and time analysis of a robotically fabricated concrete wall. Automation in Construction 92 (2018). DOI 10.1016/j.autcon.2018.04.004

46. Tang, P., Huber, D., Akinci, B., Lipman, R., Lytle, A.: Automatic reconstruction of as-built building information models from laser-scanned point clouds: A review of related techniques. Automation in Construction 19(7), 829-843 (2010). DOI 10.1016/j.autcon.2010.06.007. URL http://dx.doi.org/10.1016/j.autcon.2010.06.007

47. Thoma, A., Adel, A., Helmreich, M., Wehrle, T., Gramazio, F., Kohler, M.: Robotic Fabrication of Bespoke Timber Frame Modules. RobArch 2018 (August 2018) (2019). DOI 10.1007/978-3-319-92294-2. URL http://link.springer.com/10.1007/978-3-319-92294-2 
48. Vasey, L., Baharlou, E., Dörstelmann, M., Koslowski, V., Prado, M., Schieber, G., Menges, A., Knippers, J.: Behavioral Design and Adaptive Robotic Fabrication of a Fiber Composite Compression Shell With Pneumatic Formwork. Proceedings of ACADIA 2015 (April 2016), 297-309 (2015)

49. Vasey, L., Maxwell, I., Pigram, D.: Adaptive Part Variation: A Near Real-Time Approach to Construction Tolerances. In: W. McGee, M.P. de Leon (eds.) Robotic Fabrication in Architecture, Art and Design 2014, pp. 291-304. Springer International Publishing Switzerland 2014 (2014). DOI 10.1007/978-3-319-04663-1_20

50. Werfel, J., Petersen, K., Nagpal, R.: Designing Collective Behavior in a TermiteInspired Robot Construction Team. Science 343(6172), 754-758 (2014). DOI $10.1126 /$ science. 1245842

51. Willmann, J., Knauss, M., Bonwetsch, T., Apolinarska, A.A., Gramazio, F., Kohler, M.: Robotic timber construction - Expanding additive fabrication to new dimensions. Automation in Construction 61, 16-23 (2016). DOI 10.1016/j.autcon.2015.09.011

52. Zhang, X., Li, M., Lim, J.H., Weng, Y., Tay, Y.W.D., Pham, H., Pham, Q.C.: Large-scale 3D printing by a team of mobile robots. Automation in Construction 95(August), 98-106 (2018). DOI 10.1016/j.autcon.2018.08.004. URL https://doi.org/10.1016/j.autcon.2018.08.004 